\title{
A stable XFEM formulation for multi-phase problems enforcing the accuracy of the fluxes through Lagrange multipliers
}

\author{
P. Díez R. Cottereau and S. Zlotnik
}

\begin{abstract}
When applied to diffusion problems in a multiphase setup, the popular XFEM strategy suffers from an inaccurate representation of the local fluxes in the vicinity of the interface. The XFEM enrichment improves the global quality of the solution but it is not enforcing any local feature to the fluxes. Thus, the resulting numerical fluxes in the vicinity of the interface are not realistic, in particular when conductivity ratios between the different phases are very high. This paper introduces an additional restriction to the XFEM formulation aiming at properly reproducing the features of the local fluxes in the transition zone. This restriction is implemented through Lagrange multipliers, and the stability of the resulting mixed formulation is tested satisfactorily through the Chapelle-Bathe numerical procedure. Several examples are presented and the solutions obtained show a spectacular improvement with respect to the standard XFEM.
\end{abstract}

\section{INTRODUCTION}

Multiphase problems appear in about all fields of physics and mechanics. The classical approach to solve these problems with the Finite Element method (FEM) consists in constructing a mesh that follows the interface. Therefore, each element of the mesh pertains to only one phase, and the solution of the coupled problem can be represented reasonably well by a polynomial approximation over each element. However, when the interface is moving, the constraint that the mesh should follow the interface means that the mesh must be reconstructed at each evolution step, which can rapidly become very costly. This problem is central in metal forging, oceanography, imaging, flame modeling, melting of materials, among other applications.

Similar concerns appear for single-phase problems with intricate geometries that are solved using the immersed boundary method [1, 2, 3], or similar techniques. In these approaches, a virtual interface is created where the boundary of the domain lied, while giving very stiff or soft properties to the newly-introduced material, depending on the type of boundary condition desired. This virtual 
two-phase problem is equivalent to the original, single-phase, problem, but the meshing constraints are relaxed. This set of methods is becoming very interesting, concurrently with the ever-widening use of real microscopy images for the definition of the geometry of computational problems [4].

In the two sets of problems described above (evolving multi-phase problems and geometrically intricate single-phase problems), the possibility that the elements of the mesh be intersected by the interface is very appealing. Using the FEM, it is technically possible to do so $[5,6]$. However, the rate of convergence of the solution with respect to the size of the elements is heavily deteriorated [7]. Authors have therefore proposed alternative approaches. Among those, the Generalized and eXtended Finite Element Methods (GFEM/XFEM) have been widely developed $[8,9,10,11,12,13,14,15,16,17,18,19,20,21]$. In these methods, the classical FEM basis, using polynomials over each element, is enriched with functions that incorporate information about the interface. In the particular problems considered here, the enrichment functions introduce the possibility for the solution to have a discontinuous gradient over the interface. The improvement of the XFEM solution is often dramatic in terms of global errors, with respect to a FEM solution. However, large errors in the evaluation of the fluxes close to the interface can arise, in particular when the conductivity ratio between the two phases is large. Unfortunately, these fluxes are often very important in practice. In particular, they often provide the main drive for the evolution of the interface [22]. The main objective of this paper is to propose a method to improve this flux evaluation in the vicinity of the interface, while retaining the advantages of using unfitted meshes.

Other authors have considered similar questions. In [23], the authors considered enriched functions that consisted of the restriction of the classical finite element functions to each side of the interface. As these functions are not continuous over the interface, a variant of Nitsche's approach was used to weakly enforce the continuity of the primal variable. The main drawback of this formulation is that it involves a parameter that must be chosen with care in order to obtain a stable formulation. Nevertheless, several papers [24, 25] elaborated on variants of this original paper. Alternatively, some authors tried to enforce the continuity of the displacements through Lagrange multipliers [26]. The drawback of that approach is that it introduces additional variables in the elements cut by the interface. Also, care must be taken in the choice of the space of Lagrange multipliers in order to enforce stability. Several choices of Lagrange multiplier spaces have been proposed [27, 28], with the corresponding stability tested through numerical procedures [29, 30]. A mortar-like Lagrange approach was also proposed [31] to allow for an independent discretization of the bulk and interface fields. Note that, to the best of our knowledge, all authors use enrichment functions that are discontinuous in both the displacement and the gradient. The enrichment functions that we use in this paper are discontinuous in the gradient but continuous in the displacement (see section 3).

Although it is not central to the issues addressed in this paper, we would lastly like to comment on the parameterization of the interface. Several methods have been proposed [32], among which front tracking methods [33] and the 
marker-in-cell method [34, 35]. We consider here level-set functions [36, 37], which provide a very efficient and elegant alternative to the previous parameterizations. Since their first use in the description of dynamical two-phase fluid systems [38, 39], their power has been acknowledged for the parameterization of complex evolving phases. In particular, their ability to deal with changes in topology without any remeshing has been recognized [40, 41]. They have been used in several fields of geophysics and geomechanics, including modeling of two-phase flows and permeability estimation in reservoir simulations [41, 42], tectonic plates subduction [43, 44], seismic waves travel time computation [45], and, generally, for inverse problems and optimal design [46, 47, 48].

As stated earlier, the objective of this paper is to propose a method to improve the flux evaluation in the vicinity of the interface, while retaining the advantages of using unfitted meshes in the context of the XFEM. This paper builds on [49], where the continuity of the gradients across the interface was enforced in a strong form. The resulting formulation was unfortunately very dependent on the type of elements that were used, and no stability analysis was possible. The general form of the mixed weak formulation presented at section 5 was then briefly sketched in [50], but without the stability analysis and examples presented here. The adopted methodology is based in enforcing continuity of the flux across the interface using Lagrange multipliers. Consequently, the number of unknowns is increased with the dimension of the Lagrange multipli-

ers space. Being the interface in a manifold of lesser order with respect to the computational domain, the cost increase is considered to be very moderate. In any case, the payoff in local accuracy worths the computational effort.

The outline of the paper is the following: in section 2, the problem of interest is stated; in section 3, the XFEM and the enrichment functions that we use are discussed; in section 4, an illustration of the lack of accuracy of the XFEM for the fluxes in the vicinity of the interface is presented; in section 5 and 6 , which constitute the core of the paper, our Lagrange-based mixed formulation is introduced and its stability is discussed; and finally, in section 7, several examples illustrate the behavior of the method proposed.

\section{PROBLEM STATEMENT}

Let us consider an open bounded domain $\Omega \subset \mathbb{R}^{2}$, partitioned into two subdomains $\Omega_{1}$ and $\Omega_{2}$ with different physical characteristics (see figure 1 for notations). The boundary $\partial \Omega$ of the global domain $\Omega$ is divided into two parts on which two different types of boundary conditions (Dirichlet or Neumann) will be applied: $\partial \Omega=\bar{\Gamma}_{N} \cup \bar{\Gamma}_{D}$, with $\Gamma_{N} \cap \Gamma_{D}=\emptyset$. The interface between the two subdomains $\Omega_{1}$ and $\Omega_{2}$ is denoted $\Gamma=\left(\partial \Omega_{1} \cup \partial \Omega_{2}\right) \backslash \partial \Omega$.

We consider the following problem, which could for instance model the tem- 


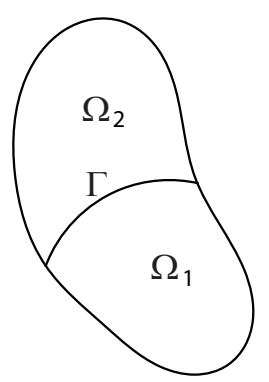

(a)

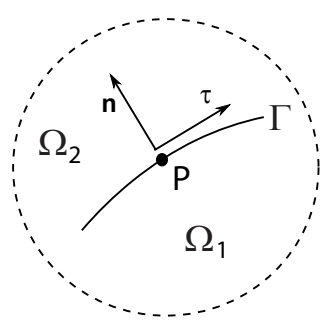

(b)

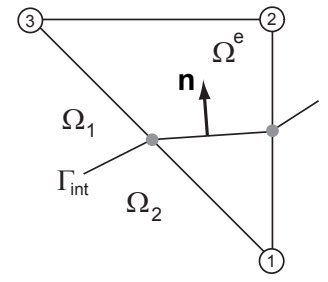

(c)

Figure 1: Illustration of (a) the complete domain $\Omega$, the subdomains $\Omega_{1}$ and $\Omega_{2}$ and the interface $\Gamma$; (b) zoom on a generic point $P$ of the interface $\Gamma$, with the definition of the normal and tangent vectors; and (c) description of the interface within an element.

perature distribution over a bi-phasic material: find $u$ such that:

$$
\begin{aligned}
-\nabla \cdot \boldsymbol{q} & =f & & \text { in } \overline{\Omega_{1}} \cup \overline{\Omega_{2}} \\
\boldsymbol{q} & =\nu \nabla u & & \text { in } \overline{\Omega_{1}} \cup \overline{\Omega_{2}} \\
\boldsymbol{q} \cdot \boldsymbol{n} & =g_{N} & & \text { on } \Gamma_{N} \\
u & =u_{D} & & \text { on } \Gamma_{D} \\
\llbracket \boldsymbol{q} \rrbracket \cdot \boldsymbol{n} & =0 & & \text { on } \Gamma \\
\llbracket u \rrbracket & =0 & & \text { on } \Gamma
\end{aligned}
$$

In these equations, $\nu=\nu_{1}$ in the phase $\Omega_{1}$ and $\nu=\nu_{2}$ in the phase $\Omega_{2}$. The normal vector $\boldsymbol{n}$ is the outgoing normal vector along $\Gamma_{N} \cup \Gamma_{D}$ and is oriented (arbitrarily) as indicated on figure 1(b), from $\Omega_{1}$ towards $\Omega_{2}$.

Note that the continuity of the (normal) flux, enforced by equation (1e), and the fact that the material coefficients $\nu_{1}$ and $\nu_{2}$ are different, implies that the gradient of $u$ is necessarily discontinuous (in the direction of the normal to $\Gamma$ ), that is

$$
\left.\nabla u\right|_{\Omega_{1}} \cdot \boldsymbol{n} \neq\left.\nabla u\right|_{\Omega_{2}} \cdot \boldsymbol{n} .
$$

Note also that the jump of the normal component of the gradient depends on the contrast between $\nu_{1}$ and $\nu_{2}$. On the contrary, the tangential component of the gradient is continuous, due to the continuity of $u$ in $\Omega$. Thus, denoting by $\tau$ the unit vector tangent to $\Gamma$ at $P$ (see figure $1(b)$ ), one has

$$
\left.\nabla u\right|_{\Omega_{1}} \cdot \boldsymbol{\tau}=\left.\nabla u\right|_{\Omega_{2}} \cdot \boldsymbol{\tau} .
$$




\subsection{Variational form}

The following functional spaces are introduced to properly state the weak form of the problem. The space $V$ containing the solution $u$ is defined as

$$
V:=\left\{u \in \mathcal{H}^{1}(\Omega): u=u_{D} \text { in } \Gamma_{D}\right\},
$$

and the corresponding test functions space, $V_{0}$ is

$$
V_{0}:=\left\{u \in \mathcal{H}^{1}(\Omega): u=0 \text { en } \Gamma_{D}\right\} .
$$

Thus, the weak form of the problem, equivalent to (1) reads: find $u \in V$ such that

$$
a(u, v)=\ell(v), \quad \text { for all } v \in V_{0},
$$

where the bilinear and linear forms $a(\cdot, \cdot)$ and $\ell(\cdot)$ are given by

$$
a(u, v):=\int_{\Omega_{1} \cup \Omega_{2}} \nu \nabla u \cdot \nabla v d \Omega \text { and } \quad \ell(v):=\int_{\Omega} f v d \Omega-\int_{\Gamma_{N}} g_{N} v d s .
$$

It is worth noting that the continuity constraint (1e) is implicitly imposed in (6). In fact, this condition is enforced in (6) in a weak fashion, in the same manner as the Neumann boundary conditions (1c). Therefore, this restriction is exactly fulfilled only if the equation is solved exactly, using the infinitedimensional spaces $V$ and $V_{0}$. In the FEM solution, using finite-dimensional spaces approximating $V_{H}$ and $V_{H_{0}}$, these conditions are only verified approximately. We come back to this issue in section 5 .

\section{PHASE TRACKING WITH LEVEL SETS AND XFEM ENRICHMENT}

In this section, we briefly recall the definitions of level sets, of the FEM, and of the XFEM, with a particular emphasis on the type of enrichment functions that are used in our version of the XFEM.

\subsection{Level sets}

Level set functions $[36,37$ provide a very efficient and elegant parameterization of multi-phases domains. In the simplest setting, they allow to discriminate between two areas of a domain, with no explicit parameterization of the actual interface. Conceptually, they are constructed in a space of higher dimension than the interface they intend to represent, with a smoother topological behavior that allows for an easier manipulation.

The level set is a function $\phi(x)$ defined over the entire domain $\Omega$, and its sign indicates the belonging to one or the other of the two areas $\Omega_{1}$ and $\Omega_{2}$ :

$$
\phi(x)= \begin{cases}>0, & x \in \Omega_{1} \\ =0, & x \in \Gamma \\ <0, & x \in \Omega_{2}\end{cases}
$$


Usually, its absolute value is defined as the distance to the interface. The interface is hence parameterized by the set of zeros of the level set.

\subsection{Finite Element Method (FEM)}

A finite element mesh generates a discrete functional space $V_{H} \subset V$, where $H$ stands for the characteristic size of the elements in the mesh. The mesh is a partition of the domain $\Omega$ into disjoint elements $\Omega^{e}, e=1, \ldots, n_{e}$, that is $\bar{\Omega}=\bigcup_{e} \bar{\Omega}^{e}$ and $\Omega^{e} \cap \Omega^{e^{\prime}}=\emptyset$ for $e \neq e^{\prime}$. The corresponding discrete counterpart of the test space $V_{0}$ is denoted by $V_{H_{0}}$. The standard FEM basis of shape functions, generating $V_{H_{0}}$ is denoted by $N_{i}, i=1, \ldots, n_{H}$, being $n_{H}$ the number of nodal points in the finite element mesh.

The affine space $V_{H}$ is therefore $V_{H}=\left\{u_{D}^{\star}\right\}+V_{H_{0}}$, where $u_{D}^{\star}$ is a function in $\operatorname{span}\left\{N_{1}, \ldots, N_{\text {poin }}\right\}$, defined over $\bar{\Omega}$ and that coincides with $u_{D}$ on $\Gamma_{D}$. Typically, $u_{D}^{\star}$ is determined as the interpolation of $u_{D}$ with the FEM mesh. In the following, it is assumed that the FEM discretization properly reproduces $u_{D}$ (no oscillation terms in the data are considered). Thus, the FEM solution $u_{H}$ is defined by the nodal values $u_{i}, i=1, \ldots, n_{H}$ :

$$
u_{H}=\sum_{i=1}^{n_{H}} N_{i} u_{i}
$$

and is such that

$$
a\left(u_{H}, v\right)=\ell(v) \text {, for all } v \in V_{H_{0}} \subset V_{0} .
$$

The discretization results in an algebraic system of equations

$$
\mathbf{K}_{u u} \boldsymbol{u}=\mathbf{F}_{u},
$$

where the unknown column vector $\boldsymbol{u}$ contains the coefficients $u_{i}$, associated to the nodes that are not on the Dirichlet boundary $\Gamma_{D}$, and the stiffness matrix $\mathbf{K}_{u u}$ and force vector $\mathbf{F}_{u}$ have generic components $\left[\mathbf{K}_{u u}\right]_{i j}=a\left(N_{i}, N_{j}\right)$ and $\left[\mathbf{F}_{u}\right]_{j}=\ell\left(N_{j}\right)-a\left(u_{D}^{\star}, N_{j}\right)$.

A consequence of the assumption of no data oscillations is that the error associated with $u_{H}, e:=u-u_{H}$, belongs to $V_{0}$. Thus, Galerkin orthogonality holds and $u_{H}$ is optimal in the sense that

$$
\left\|u-u_{H}\right\| \leq\|u-v\| \quad \forall v \in V_{H}
$$

where $\|\cdot\|$ is the energy norm, defined by $\|v\|^{2}=a(v, v)$.

The level set function $\phi$ is also approximated by $\phi_{H} \in V_{H}$ and determined by the nodal values $\phi_{i}, i=1, \ldots, n_{H}$,

$$
\phi \approx \phi_{H}=\sum_{i=1}^{n_{H}} N_{i} \phi_{i} .
$$


This allows to describe the interface as a continuous line (surface in 3D), smooth inside the elements and with slope discontinuities when intersecting the element edges (sides in $3 \mathrm{D}$ ). For linear triangular elements the interface is a polygonal line.

Thus, the normal vector to the interface (see Figure 1(c)) is easily computed at any point of the interface in the interior of an element $\Omega^{e}$ :

$$
\mathbf{n}=\frac{\nabla \phi_{H}}{\left\|\nabla \phi_{H}\right\|} \quad \text { with } \quad \nabla \phi_{H}=\sum_{i=1}^{n_{H}} \phi_{i} \nabla N_{i} .
$$

Note that, as $\phi_{H}$ is positive on $\Omega_{1}$, its gradient, and consequently $\mathbf{n}$, point from $\Omega_{1}$ towards $\Omega_{2}$.

\subsection{EXtended Finite Element Method (XFEM)}

The standard FEM provides a solution $u_{H}$ that is infinitely regular (smooth) inside any element $\Omega^{e}$. Therefore, standard finite elements are unable to reproduce the gradient jumps of the actual solution (see (1e) and (2)). The XFEM enriches the FEM solution using a ridge function, hence enabling the discretization to introduce a jump of the gradient across the interface. The ridge function used here is the one introduced in [15] and defined as

$$
R=\sum_{i=1}^{n_{H}} N_{i}\left|\phi_{i}\right|-\left|\sum_{i=1}^{n_{H}} N_{i} \phi_{i}\right|
$$

The function $R$ vanishes in all the elements that are not crossed by the interface. Thus, the support of $R$ is precisely the set of elements that should be enriched. In the following, the set of indices of these elements is denoted by $\mathcal{E}_{a}$. Introducing $\mathcal{N}_{a}$ as the set of nodal indices corresponding to the nodes belonging to the enriched elements, the previous expression is rewritten as

$$
R=\sum_{i \in \mathcal{N}_{a}} N_{i}\left|\phi_{i}\right|-\left|\sum_{i \in \mathcal{N}_{a}} N_{i} \phi_{i}\right|
$$

For the sake of a simpler notation and without any loss in generality, it is assumed that the enriched nodes are the first in the node list, that is $\mathcal{N}_{a}=$ $\left\{1,2, \ldots\right.$, card $\left.\mathcal{N}_{a}\right\}$. Thus, the XFEM approximation, $u_{X}$, is

$$
u_{X}=\sum_{i=1}^{n_{H}} N_{i} u_{i}+\sum_{j \in \mathcal{N}_{a}} R N_{j} a_{j}
$$

where the coefficients $a_{j}, j \in \mathcal{N}_{a}$, stand for the enriched nodal coefficients.

The approximation $u_{X}$ lies in the discrete functional space $V_{X}=V_{H} \oplus$ $\operatorname{span}\left\{R N_{i}, i \in \mathcal{N}_{a}\right\}$ and fulfills an equation analogous to (10) but replacing $V_{H}$ by $V_{X}$. The resulting algebraic linear system of equations reads

$$
\left[\begin{array}{ll}
\mathbf{K}_{u u} & \mathbf{K}_{u a} \\
\mathbf{K}_{u a}^{\top} & \mathbf{K}_{a a}
\end{array}\right]\left[\begin{array}{l}
\boldsymbol{u} \\
\boldsymbol{a}
\end{array}\right]=\left[\begin{array}{l}
\boldsymbol{F}_{u} \\
\boldsymbol{F}_{a}
\end{array}\right]
$$


where $\boldsymbol{a}$ is the column vector of unknowns $a_{j}, j \in \mathcal{N}_{a}$ and the generic entries of the matrices and vectors are $\left[\mathbf{K}_{u a}\right]_{i j}=a\left(N_{i}, R N_{j}\right),\left[\mathbf{K}_{a a}\right]_{i j}=a\left(R N_{i}, R N_{j}\right)$, and $\left[\mathbf{F}_{a}\right]_{j}=\ell\left(R N_{j}\right)$.

Similarly to the FEM solution $u_{H}, u_{X}$ is optimal from an energetic point of view in the sense that Galerkin orthogonality stands in $V_{X}$. Consequently, the error $u-u_{X}$ is such that

$$
\left\|u-u_{X}\right\| \leq\|u-v\|, \quad \forall v \in V_{X} .
$$

Note that, taking $v=u_{H} \in V_{H} \subset V_{X}$ yields

$$
\left\|u-u_{X}\right\| \leq\left\|u-u_{H}\right\| .
$$

\section{FLUX APPROXIMATION ON THE INTER- FACE}

As previously stated, the FEM is unable to fulfill the flux continuity (1e) at the points on the interface in the interior of the elements. This is because the gradient of $u_{H}$ is continuous inside the elements and therefore equation (2) cannot be fulfilled at these points. Recall that the continuity of the normal flux at a point $P$ of the interface reads

$$
\left(\boldsymbol{q}_{2} \cdot \mathbf{n}_{2}+\boldsymbol{q}_{1} \cdot \mathbf{n}_{1}\right)=\left(\boldsymbol{q}_{2}-\boldsymbol{q}_{1}\right) \cdot \mathbf{n}=\left(\nu_{2} \nabla u_{H}-\nu_{1} \nabla u_{H}\right) \cdot \mathbf{n}=0 .
$$

However, the gradient of the standard finite element approximation, $\nabla u_{H}$, is continuous inside the elements, that is

$$
\left(\nu_{2} \nabla u_{H}-\nu_{1} \nabla u_{H}\right) \cdot \mathbf{n}=\left(\nu_{2}-\nu_{1}\right) \nabla u_{H} \cdot \mathbf{n} .
$$

Thus, the continuity of the flux cannot be achieved with standard finite elements unless the gradient (and the flux) is identically zero. This is not the case in a general situation. Further, it should be noted that the approximation deviates especially far from the exact solution when the contrast between the two coefficients $\nu_{1}$ and $\nu_{2}$ increases.

The XFEM uses an enriched basis that allows jumps in the normal component of the gradient of the solution $\nabla u_{H}$. Hence, it is expected that it may give $u_{H}$ the additional freedom to verify the flux continuity (17). Following equation (17), the gradient of the approximation can be written:

$$
\nabla u_{X}=\sum_{i=1}^{n_{H}} u_{i} \nabla N_{i}+\sum_{j \in \mathcal{N}_{a}}\left(R \nabla N_{j}+N_{j} \nabla R\right) a_{j} .
$$

The possible discontinuity of $\nabla u_{X}$ in a point $P$ of $\Gamma$, in the interior of an element $\Omega^{e}$, appears thanks to the term $\nabla R$. Indeed, $\nabla N_{i}$ and $R$ are both continuous inside $\Omega^{e}$. Further, since $\phi$ has been assumed positive inside the domain $\Omega_{1}$ and negative inside $\Omega_{2}$ (by convention, see equation (8)), and thanks to the 
definition of $R$ in equation (16), we have the following expressions for $R$ on both sides of the interface:

$$
\left.R\right|_{\Omega_{1}}=\sum_{i \in \mathcal{N}_{a}} N_{i}\left(\left|\phi_{i}\right|-\phi_{i}\right) \text { and }\left.R\right|_{\Omega_{2}}=\sum_{i \in \mathcal{N}_{a}} N_{i}\left(\left|\phi_{i}\right|+\phi_{i}\right) .
$$

Note that $\left.\nabla R\right|_{\Omega_{2}}-\left.\nabla R\right|_{\Omega_{1}}=2 \nabla \phi_{H} \neq 0$, where $\phi_{H}$ denotes the level set function, as defined in equation (13). As expected, the jump in the gradient of $R$ is parallel to the normal $\boldsymbol{n}$, since the latter is parallel to $\nabla \phi_{H}$. Thanks to this additional freedom with respect to the classical finite element approximation, it is expected that the XFEM approximation may perform substantially better than the FEM.

In the rest of this section, we illustrate the behavior of the XFEM in terms of global error and quality of the approximation of the fluxes in the vicinity of the interface. We therefore present two simple examples: a square with a horizontal interface (intersecting the elements of the mesh), and the same with a tilted interface. In the first example, the XFEM captures the exact solution while the FEM behaves poorly. In the second example, the XFEM behaves well in terms of global error, but the fluxes are wrongly evaluated.

Exemple 1a (horizontal interface): Let us consider the problem (1), with the following parameters: $\Omega=[-1,1] \times[-1,1], g_{N}=0$ for $x= \pm 1, u_{D}=1$ for $y=1, u_{D}=0$ for $y=-1, f=0, \nu_{1}=1000$ and $\nu_{2}=1$. Note that the ratio of conductivities is very large. We first consider a perfectly horizontal interface, at $y=0.1$ (cutting the elements of the proposed mesh), with $\Omega_{2}$ at $y>0.1$. In this first configuration, the problem is quasi-1D. The exact solution is:

$$
u(x, y)=\frac{1}{1100,9}\left\{\begin{array}{ll}
1000(y+1) & \text { for } y \leq 0.1 \\
y+1099,9 & \text { for } y>0.1
\end{array} .\right.
$$

Since the solution is linear, if the mesh were to follow the interface, the FEM solution would be the exact one. However, in the case that we are interested in, the interface goes through the elements, and we can observe on figure 2 that the solution in terms of fluxes is rather inaccurate. More precisely, because the gradient of the FEM solution is continuous and the contrast in conductivities is very large, the fluxes appear to almost cancel on one side of the interface in the elements that are cut. Hence it appears that there are two jumps in the fluxes: one over the interface, created purely by jump in conductivities; and one between the line of elements cut by the interface and the next one, only on the weak side (where $\nu=1$ ). On the other side, this discontinuity between the elements cut by the interface and the first line perfectly included inside a phase is not so obvious. These last two remarks are related to the fact that, because it is not using a functional basis that incorporates information on the interface, the FEM effectively sees an average value of the conductivity rather than two phases. As the contrast if very large, this average conductivity is close to $\nu_{1}=1000$, even for elements almost entirely contained in $\Omega_{2}$. Finally, it is important to understand that this local quantity of interest will not necessarily 


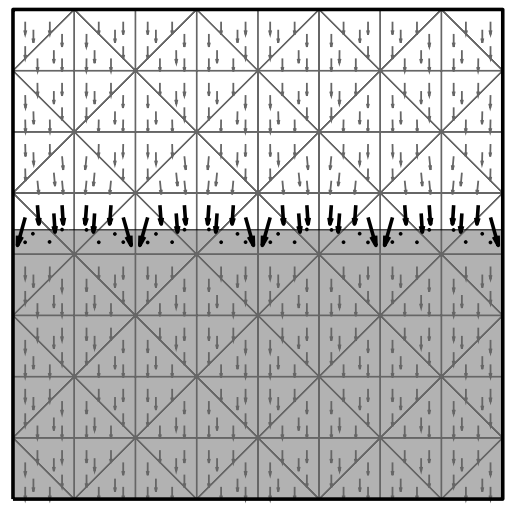

(a) FEM solution, $\left\|u_{\mathrm{FEM}}\right\|=1.997$

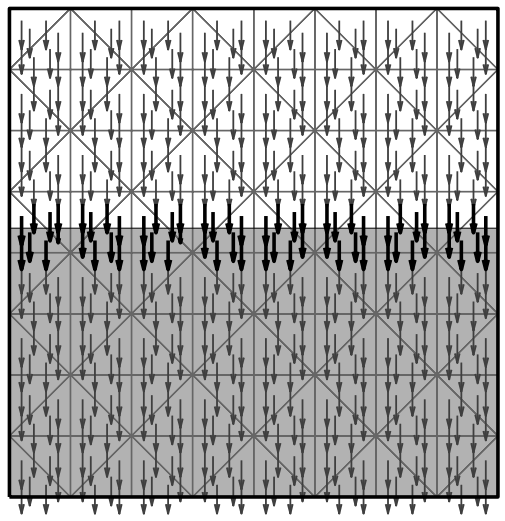

(b) XFEM solution, $\left\|u_{\text {XFEM }}\right\|=1.816$

Figure 2: Example 1a (horizontal interface): the XFEM (right figure) performs better than the FEM (left figure) both in terms of global energy norm and local fluxes. The fluxes are evaluated at the Gauss points of each element and drawn in bold for the elements that are cut by the interface.

be better estimated with a more refined mesh. The global approximation will become better, but very locally, these two discontinuities will still be observed. On this first configuration, on the other hand, the XFEM captures the analytical solution. Indeed, the enriched basis includes the exact solution. Note that the comparison between FEM and XFEM is fairly performed in terms of the approximation properties of the discrete functional spaces. The integration of the elementary matrices and vectors is therefore performed using the same quadrature. In that sense, the version of FEM used here is nonstandard because it also splits the multiphase elements for integration purposes in order to obtain the stiffness matrix and the force vectors with the same accuracy as the ones typically obtained with XFEM.

Example 1b (tilted interface): We now consider the same problem as before, but with a slightly tilted interface $\left(\right.$ slope $-15^{\circ}$ ). In that case, the solution is really 2D, and is plotted in Figure 3. As before, the FEM solution for a mesh that does not follow the interface is a rather poor approximation of the exact solution (computed using the FEM with a fitted mesh), both in terms of global energy norm and local evaluation of fluxes in the vicinity of the interface. Again, the XFEM behaves well in terms of global energy norm, practically coinciding with the exact energy, see [51] for analytical expressions of the same type of problems. However, this time, the XFEM seems to behave as bad as the FEM in terms of fluxes. This is due to the fact that the gradient of the solution may indeed be discontinuous, but that the chosen degrees of freedom, and the 


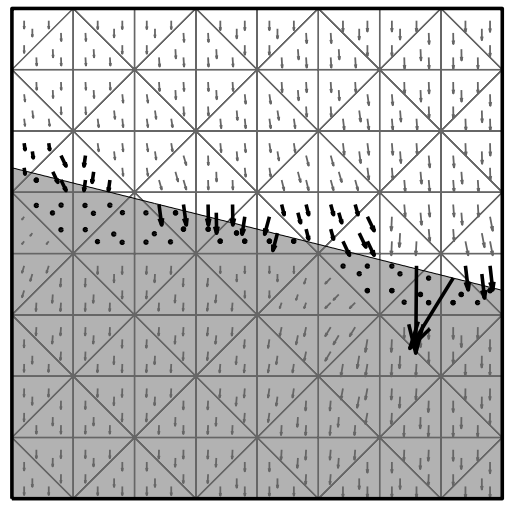

(a) FEM solution, $\left\|u_{\text {FEM }}\right\|=2.237$

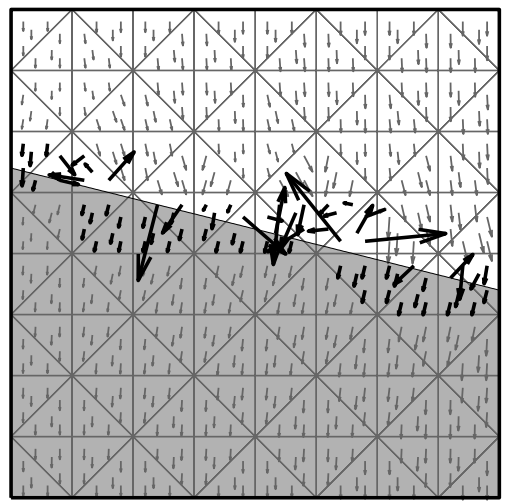

(b) XFEM solution, $\left\|u_{\text {XFEM }}\right\|=1.892$

Figure 3: Example 1b (tilted interface): the additional degrees of freedom in the XFEM (right figure) induce an improvement with respect to the FEM solution (left figure), in terms of the global energy norm, but not necessarily an improved evaluation of the fluxes close to the interface.

corresponding functional basis, do not allow to fix the gradients independently on each side of the interface. There is an additional freedom with respect to the FEM, but the exact solution is not necessarily contained in the approximating functional space. When the contrast in conductivity becomes large, large variations in the fluxes close to the interface can happen with small variations of the mesh.

\section{$5 \quad$ Explicitly Enforcing Flux Continuity}

We come back in this section to the way the flux continuity is enforced in the FEM and XFEM and propose to enforce it explicitly to improve the numerical results in terms of fluxes.

\subsection{Weak approximation of Neumann Boundary Condi- tions and flux continuity on the interface}

The interface condition (1e) is weakly enforced, in the same fashion as the Neumann B.C. (1c). Recall that, when integrating by parts the weighted residual of (1a), the term including the flux on $\Gamma_{N}$,

$$
\int_{\Gamma_{N}} \nu \nabla u \cdot \mathbf{n} v d s \quad \text { is replaced by } \quad \int_{\Gamma_{N}} g_{N} v d s
$$


using (1c). In a similar way, integrating by parts in $\Omega_{1}$ and $\Omega_{2}$ and adding two expressions, a flux jump term appears in the integral form

$$
\int_{\Gamma}\left(\boldsymbol{q}_{2}-\boldsymbol{q}_{1}\right) \cdot \mathbf{n} v d \Gamma
$$

that is readily taken as 0 attending to (1e). The numerical flux on $\Gamma_{N}$ converges asymptotically to $g_{N}$ as $h$ tends to zero. The discrete approximation of the fluxes is such that equilibrium is reached in the discrete form, for the sum of the nodal versions of the body loads $f$ and the surface loads $g_{N}$. Thus, for a given mesh, the numerical flux on $\Gamma_{N}$ may be a poor approximation to $g_{N}$, that is, it does not fit the data. The same occurs with the flux jump on $\Gamma$ and, consequently, with the values of $\boldsymbol{q}_{1} \cdot \mathbf{n}$ and $\boldsymbol{q}_{2} \cdot \mathbf{n}$ which are, in practice, far from being accurate approximations.

\subsection{Enforcing the flux continuity explicitly}

In order to improve the quality of the fluxes on $\Gamma$, we propose to enforce explicitly the weak condition of continuity. That is, we propose to add the following condition to the XFEM solution, $u_{X}$,

$$
b\left(\mu, u_{X}\right)=0, \text { for all } \mu \in \tilde{V}_{H},
$$

where $\tilde{V}_{H}$ is a discrete space of weighting functions, that has to be chosen, and

$$
b(\mu, u):=\int_{\Gamma}\left(\boldsymbol{q}_{2}-\boldsymbol{q}_{1}\right) \cdot \mathbf{n} \mu d \Gamma=\int_{\Gamma}\left(\left.\nu_{2} \nabla u\right|_{\Omega_{2}}-\left.\nu_{1} \nabla u\right|_{\Omega_{1}}\right) \cdot \mathbf{n} \mu d \Gamma .
$$

Note that the weighting functions $\mu$ should belong to $L^{2}(\Gamma)$.

With this additional constraint, the flux continuity (1e) is expected to be fulfilled more accurately because it is not anymore competing with the residual coming from the interior of the domain.

The condition (27) is added to the original weak form (6) (or its discrete counterpart (10)) using the Lagrange multipliers approach. Thus, the resulting mixed problem reads: find $u_{X} \in V_{X}$ and $\lambda_{H} \in \tilde{V}_{H}$ such that

$$
\begin{aligned}
a\left(u_{X}, w\right)+b\left(\lambda_{H}, w\right) & =\ell(w) & & \forall w \in V_{X, 0} \\
b\left(\mu, u_{X}\right) & =0 & & \forall \mu \in \tilde{V}_{H}
\end{aligned}
$$

The matrix form of (29) results in an enlarged algebraic system of linear equations

$$
\left[\begin{array}{ccc}
\mathbf{K}_{u u} & \mathbf{K}_{u a} & \mathbf{R}_{u} \\
\mathbf{K}_{u a}^{\top} & \mathbf{K}_{a a} & \mathbf{R}_{a} \\
\mathbf{R}_{u}^{\top} & \mathbf{R}_{a}^{\top} & \mathbf{0}
\end{array}\right]\left[\begin{array}{c}
\boldsymbol{u} \\
\boldsymbol{a} \\
\boldsymbol{\lambda}
\end{array}\right]=\left[\begin{array}{c}
\mathbf{F}_{u} \\
\mathbf{F}_{a} \\
\mathbf{0}
\end{array}\right]
$$

where the matrices $\mathbf{R}_{u}$ and $\mathbf{R}_{a}$ are defined below.

The Lagrange multipliers space $\tilde{V}_{H}$ must be chosen with care. If its dimension is too small, the flux continuity condition may be not be enforced strongly 


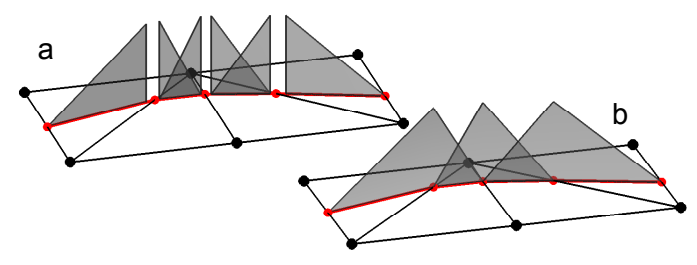

Figure 4: Representation of typical elements of the trace space of $V_{H}$ (right figure) and of the broken space $\tilde{V}_{H}$ (left figure).

enough, so that the improvements might be limited with respect to the classical XFEM. If its dimension is too large, the resulting discrete problem may become unstable.

Indeed, we have observed through numerical tests that the obvious choice of selecting $\tilde{V}_{H}$ as the trace space (over the interface $\Gamma$ ) of the FE space $V_{H}$ does not introduce enough constraints to obtain a noticeable improving of the solution. Note that for this choice $\tilde{V}_{H}$ is generated by functions as the one depicted in Figure 4 and its dimension $n_{I}$ is equal to the number of element edges crossed by the interface.

An alternative choice, already considered in [52], consists in taken $\tilde{V}_{H}$ as the broken space generated by the functions $\tilde{N}_{k}$ depicted in Figure 4 . The dimension of $\tilde{V}_{H}$ is $2 n_{I}$ because every edge crossed by the interface introduces two functions $\tilde{N}_{k}$ and $\tilde{N}_{k+1}$. For every $k=1, \ldots, 2 n_{I}$, the generic components of the matrices $\mathbf{R}_{u}$ and $\mathbf{R}_{a}$ in (30) read

$$
\left[\mathbf{R}_{u}\right]_{i k}=b\left(\tilde{N}_{k}, N_{i}\right) \text { for } i=1, \ldots, n_{H},
$$

and

$$
\left[\mathbf{R}_{a}\right]_{j k}=b\left(\tilde{N}_{k}, R N_{j}\right) \text { for } j \in \mathcal{N}_{a} .
$$

We will show in Section 6 that the corresponding discrete problem is stable.

Moreover, the flux jump on the interface is computed in a natural way in XFEM, leading to a simple computation of the Lagrange multipliers matrices $\mathbf{R}_{u}$ and $\mathbf{R}_{a}$ as

$$
\left[\mathbf{R}_{u}\right]_{i k}=\int_{\Gamma}\left(\nu_{2} \nabla N_{i}-\nu_{1} \nabla N_{i}\right) \cdot \mathbf{n} \tilde{N}_{k} d \Gamma
$$

and

$$
\left[\mathbf{R}_{a}\right]_{j k}=\int_{\Gamma}\left(\left.\nu_{2} \nabla\left(R N_{j}\right)\right|_{\Omega_{2}}-\left.\nu_{1} \nabla\left(R N_{j}\right)\right|_{\Omega_{1}}\right) \cdot \mathbf{n} \tilde{N}_{k} d \Gamma
$$

where the normal vector $\mathbf{n}$ is computed as defined in (14).

As a final remark, and as is classically done in XFEM, it should be noted that the nodes are enriched only when the value of the level set in these nodes $\left(\phi_{i}\right)$ is, in absolute value, larger than a given threshold $\epsilon$. Otherwise, the enrichment would only result in redundant parameterization. In all the computations 
performed in this paper, the threshold has been set to $\epsilon=0.05$, relative to a characteristic size of the smallest element of the mesh. On the other hand, it has been observed in the numerical experiments that normalizing functions $\tilde{N}_{k}$ in the broken space $\tilde{V}_{H}$, for instance enforcing a unitary slope as in some implementations of the classical finite elements, does not bring a substantial improvement of the condition number of the global matrix.

\section{$6 \quad$ Stability analysis}

The method of Lagrange multipliers leads to a saddle-point problem. The infsup condition (or LBB) states [53] that the resulting formulation is stable if there exists a $k>0$, independent of the mesh size, such that

$$
\inf _{\mu \in \tilde{V}_{H}} \sup _{v \in V_{X}} \frac{b(\mu, v)}{\|\mu\|_{\tilde{V}_{H}}\|v\|_{V_{X}}} \geq k
$$

Note that the two norms in the denominator are different one from the other. One is defined over the domain $\Omega$ while the other is defined over the interface $\Gamma$.

It is often difficult to prove analytically that the LBB condition is fulfilled. In appendix A, we give one sketch of such proof of stability. However, as it is based on some strong hypotheses, that are difficult to prove in practice, we concentrate in this section on the Chapelle-Bathe test [29] that consists in solving the eigenvalue problem for a series of meshes and showing the boundedness of the smallest eigenvalue. Passing the numerical test does not guarantee stability but experience shows that it is very sensitive, and indeed manages to discriminate between stable and unstable problems.

The functions $\mu(x) \in \tilde{V}_{H}$ and $v(x) \in V_{X}$ can be written as vector products $\mu(x)=\tilde{\mathbf{N}}(x)^{\top} \boldsymbol{\mu}$ and $v=\mathbf{N}(x)^{\top} \mathbf{v}$, where $\tilde{\mathbf{N}}$ and $\mathbf{N}$ are vectors of the respective interpolation functions. The length (number of terms) of vector $\mathbf{N}$ is $n_{\mathrm{x}}=$ $n_{H}+\operatorname{card} \mathcal{N}_{a}=\operatorname{dim} V_{X}$ and the length of $\tilde{\mathbf{N}}$ is $2 n_{I}$. Their generic terms read: $[\tilde{\mathbf{N}}(x)]_{i}=\tilde{N}_{i}(x)$, for $1 \leq i \leq 2 n_{I},[\mathbf{N}(x)]_{i}=N_{i}(x)$, for $1 \leq i \leq n_{H}$, and $[\mathbf{N}(x)]_{n_{H}+i}=R(x) N_{i}(x)$, for $i \in \mathcal{N}_{a}$, and where $\boldsymbol{\mu}$ and $\mathbf{v}$ are the vectors of the nodal values of the functions. The matrix form of the operator $b(\mu, v)$ in (33) can then be expressed as

$$
b(\mu, v)=\int_{\Gamma_{\text {int }}} \mu \llbracket \nu \nabla v \cdot \mathbf{n} \rrbracket d \Gamma=\boldsymbol{\mu}^{\top} \mathbf{R} \mathbf{v}
$$

where $\mathbf{R}=\left[\mathbf{R}_{u}^{T} \mathbf{R}_{a}^{T}\right]$ is a $2 n_{I} \times n_{\mathrm{x}}$ rectangular matrix, whose blocks are defined in Equation (31). Assuming that, for every possible Lagrange multiplier $\mu \in \tilde{V}_{H}$, there exists a solution $w \in V_{X}$, such that $\mu=\llbracket \nu \nabla w \cdot \mathbf{n} \rrbracket$, we introduce the matrix $\mathbf{J}$ such that $\boldsymbol{\mu}=\mathbf{J} \mathbf{w}$. This matrix $\mathbf{J}=\left[\begin{array}{ll}\mathbf{J}_{u}^{T} & \mathbf{J}_{a}^{T}\end{array}\right]$ represents the flux jump operator in the bases $\tilde{\mathbf{N}}$ and $\mathbf{N}$. The general term $[\mathbf{J}]_{i j}$ is such that $\sum_{j=1}^{2 n_{I}}\left[\mathbf{J}_{u}\right]_{i j} \tilde{N}_{j}=\llbracket \nu \nabla N_{i} \cdot \mathbf{n} \rrbracket$ for $1 \leq i \leq n_{H}$, and $\sum_{j=1}^{2 n_{I}}\left[\mathbf{J}_{a}\right]_{i j} \tilde{N}_{j}=\llbracket \nu \nabla\left(R N_{i}\right) \cdot \mathbf{n} \rrbracket$ for $i \in \mathcal{N}_{a}$. We note that

$$
\mathbf{R}=\mathbf{M}_{\mu} \mathbf{J}
$$




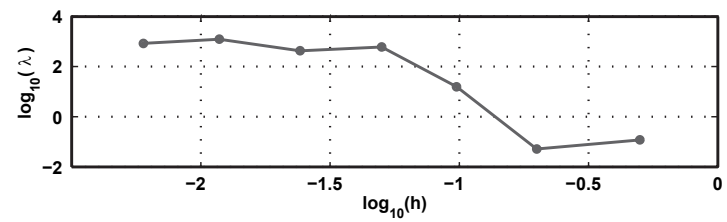

Figure 5: Chapelle-Bathe test: evolution of the smallest eigenvalue as a function of the size of the mesh.

where $\mathbf{M}_{\mu}$ is a mass matrix (corresponding to the $\mathcal{L}_{2}$ product) for the elements of $\tilde{V}_{H}$, with general term $\left[\mathbf{M}_{\mu}\right]_{i j}=\int_{\Gamma_{i n t}} \tilde{N}_{i} \tilde{N}_{j} d \Gamma$. We then obtain the discretized LBB condition:

$$
\inf _{\mathbf{w} \in \mathbb{R}^{2 n_{\mathbf{x}}}} \sup _{\mathbf{v} \in \mathbb{R}^{2 n_{\mathbf{x}}}} \frac{\mathbf{w}^{\top} \mathbf{J}^{\top} \mathbf{M}_{\mu} \mathbf{J} \mathbf{v}}{\left(\mathbf{w}^{\top} \mathbf{J}^{\top} \mathbf{M}_{\mu} \mathbf{J} \mathbf{w}\right)^{1 / 2}\left(\mathbf{v}^{\top} \mathbf{M}_{v} \mathbf{v}\right)^{1 / 2}} \geq k
$$

where $\mathbf{M}_{v}$ is the mass matrix for $V_{X}$. Note that all the norms are equivalent here, and we chose the $\mathcal{L}_{2}$ norms for both $\|\cdot\|_{\tilde{V}_{H}}$ and $\|\cdot\|_{V_{X}}$. The ChapelleBathe numerical test [29] consists in checking with numerical experiments the boundedness (from below) of the smallest non-zero eigenvalue of the generalized eigenvalue problem: find $\mathbf{w} \in \mathbb{R}^{2 n_{x}}$ and $\lambda \in \mathbb{R}$ such that

$$
\left(\mathbf{J}^{\top} \mathbf{M}_{\mu} \mathbf{J}\right) \mathbf{w}=\lambda \mathbf{M}_{v} \mathbf{w} .
$$

We therefore have to compute the smallest non-zero eigenvalue for a series of discretizations of the same domain with increasingly smaller elements. If that value does not appear to vanish for refined meshes, the test is considered passed.

We consider for the test the Example 1b, described in Section 4 (see also Figure 6 for the solution obtained with our approach). The results are plotted in Figure 5 and indeed indicate that the test is passed.

\section{Numerical examples}

The examples of Section 4 show that, even in very simple scenarios, the FEM introduces a jump in the flux across the interface. Less intuitively, these examples show that the XFEM solution presents continuous fluxes over the interface only when the flux is orthogonal to the interface (see Figures 2 and 3). In this section, we first show that our method works well for Example 1b. We then compare the behavior of our method to the FEM and XFEM solutions in the case of an unstructured grid. Finally, we describe the convergence of the solutions obtained with the three methods in terms of the norm of the flux along the interface. This convergence study is performed for a problem with analytical

solution. For easier reference in the remainder of this section, we denote our method by XFEM+. 


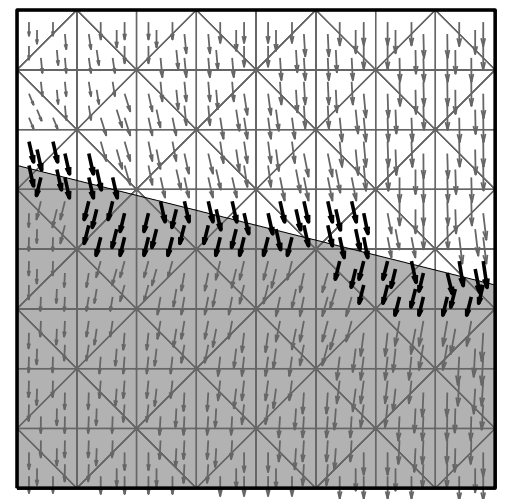

(a) XFEM+ solution, $\left\|u_{\mathrm{XFEM}+}\right\|=1.900$
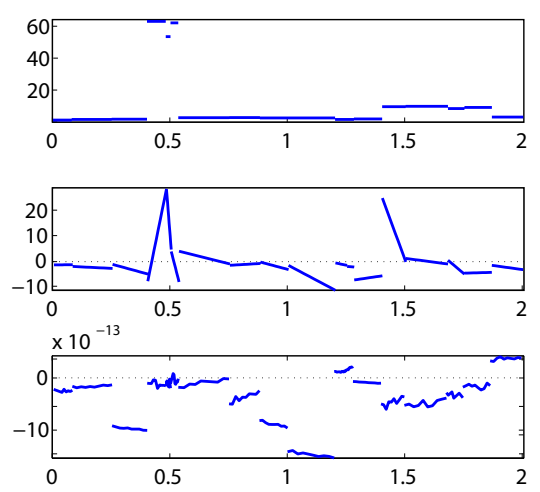

(b) Flux jump along the interface

Figure 6: Example 1b: fluxes and energy norm of the solution computed with the XFEM+ (left figure, to be compared with Figure 3) and flux jump along the interface computed with the FEM (upper right figure), the XFEM (center right figure) and the XFEM+ (lower right figure).

\subsection{Example 1b.}

We first apply the XFEM+ to Example 1b, which was already solved with the FEM and XFEM. The setting is described in Section 4 and maps of fluxes computed with the FEM and XFEM are presented in Figure 3. As illustrated in Figure 6(a), the fluxes computed with the XFEM+ are visually much more reasonable than those computed with the FEM or XFEM. Also, as expected, the flux jump cancels along the interface for the XFEM+. Note also that the flux jump is constant along the interface within one element with the FEM, while it is linear with the XFEM.

In Table 1, we present a comparison of the solution of Example 1b obtained with the three schemes. The comparison is performed both in terms of a global quantity, the energy norm, and local quantities, the maximum and mean flux jump along the interface. As explained earlier, the best solution in terms of the global energy norm is the XFEM, but the XFEM+ performs almost equally well. In terms of the local quantities of interest, however, the XFEM does not behave better than the FEM, and only the XFEM+ gives good results. In this example we analyze the value of the flux jump, rather than the actual value of the flux which could be analyzed using the reference solution for Example 1b provided in [51]. This is because we postpone this discussion to Section 7.3 in which the analytical solution is available and very simple to describe. 


\begin{tabular}{rccc}
\hline \hline scheme & FEM & XFEM & XFEM+ \\
\hline global energy norm & 2.237 & 1.892 & 1.900 \\
max flux jump & 66.3 & 37.06 & $1.84 \mathrm{e}-12$ \\
mean flux jump & 13.4 & 5.0 & $4.4 \mathrm{e}-13$ \\
\hline \hline
\end{tabular}

Table 1: Example 1b: comparison, in terms of global energy norm, maximum and minimum flux jumps (in absolute value) of the solutions obtained with the three schemes.

\begin{tabular}{rccc}
\hline \hline scheme & FEM & XFEM & XFEM+ \\
\hline global energy norm & 1.924 & 1.824 & 1.824 \\
max flux jump & 5.5 & 4.22 & $3.9 \mathrm{e}-12$ \\
mean flux jump & 2.4 & 0.5 & $1.4 \mathrm{e}-12$ \\
\hline \hline
\end{tabular}

Table 2: Unstructured mesh example: comparison, in terms of global energy norm, maximum and minimum flux jumps (in absolute value) of the solutions obtained with the three schemes.

\subsection{Unstructured meshes}

In this example, we consider the same problem as before, with two small modifications: the slope is now $-5^{\circ}$ and a non-structured mesh is used. The objective is to show that the results obtained earlier are not dependent on the specific alignment of the interface with the edges of the elements. The results obtained with the three schemes are presented in Figure 7. The fluxes in the elements crossed by the interface are plotted at the Gauss integration points, and the flux jump is plotted along the interface. The results confirm the conclusions of the previous example and imply that the method is valid whatever the relative alignment of the interface with the sides of the elements it crosses.

The proposed scheme adds restrictions to the XFEM functional space where the solution is sought. Consequently, the XFEM+ space is strictly contained inside the XFEM space. Thus, if measured in the global energy norm, the error for XFEM+ is necessarily larger than for XFEM. This is observed in the examples and shown in Table II. Nevertheless, the loss of accuracy associated with this phenomenon is very small (the energy norm of the XFEM+ error is only $0.4 \%$ larger than the XFEM one) if compared with the difference between FEM and XFEM (discrepancies of around 15\%). In other words, a little price is paid in terms of energy to improve the accuracy of the fluxes.

\subsection{Convergence study}

The previous examples indicated that the flux jumps computed by the FEM and XFEM were not correct, and showed that the fluxes evaluated with the FEM and XFEM presented variabilities not compatible with the simplicity of the problems considered. However, we have not yet shown quantitative arguments to prove that the fluxes are indeed well estimated with the XFEM + . We therefore 


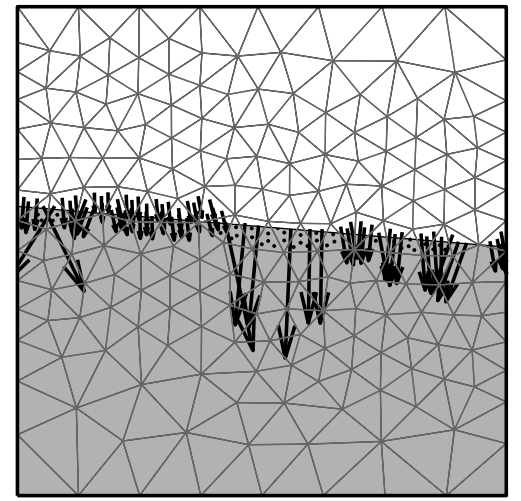

(a) FEM solution, $\left\|u_{\mathrm{FEM}}\right\|=1.924$

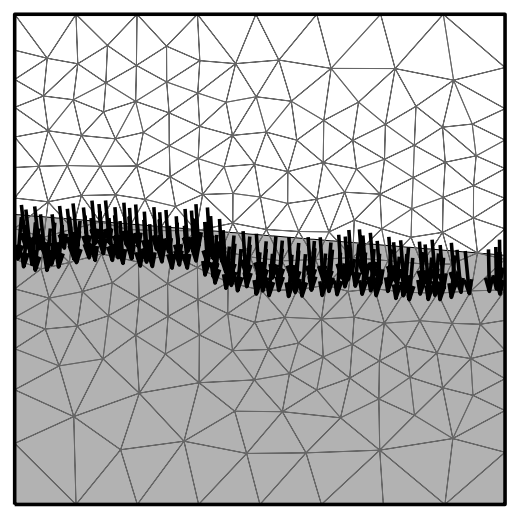

(c) XFEM+ solution, $\left\|u_{\mathrm{XFEM}}+\right\|=1.824$

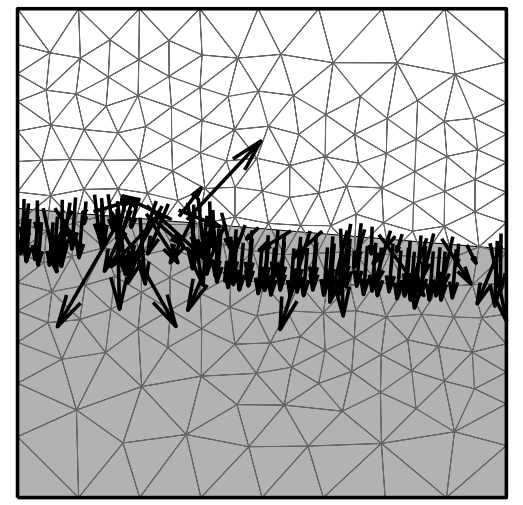

(b) XFEM solution, $\left\|u_{\text {XFEM }}\right\|=1.824$
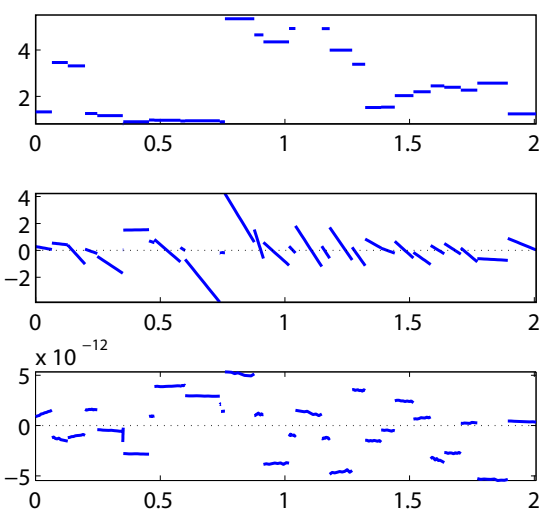

(d) Flux jump along the interface

Figure 7: Unstructured mesh example: fluxes and energy norm of the solution computed with the three schemes (figures (a), (b) and (c)) and flux jump along the interface computed with the FEM ((d), upper figure), the XFEM ((d) center figure) and the XFEM+ ((d) lower figure). 


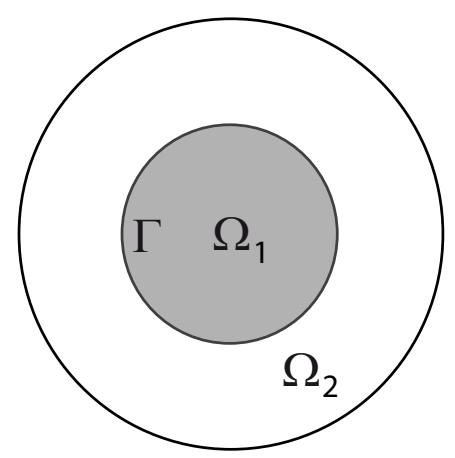

Figure 8: Discretized domain and interface for the convergence study

consider a new problem, for which an analytical solution is known, and compute errors for the three schemes, in terms of the flux over the interface.

Let us consider the open disc $\Omega \in \mathbb{R}^{2}$ centered on $(0,0)$, and with radius 1 . It is split into two concentric subdomains $\Omega_{1}$ (disc) and $\Omega_{2}$ (ring) at radius $1 / 2$ (see Figure 8). We use cylindrical coordinates $(r, \theta)$, and define the parameter $\nu_{1}=1000$ and $\nu_{2}=1$. We finally consider the following problem: find $u$ such that

$$
\begin{cases}\nu \Delta u+4=0 & , \text { in } \Omega \\ u=0 & , \text { at } r=1\end{cases}
$$

with continuity of $u$ and normal flux $q=\nu \nabla u \cdot \mathbf{e}_{r}$ at the interface $r=r_{0}$. The exact solution $u_{\text {ex }}$ of that problem is given by

$$
\begin{cases}u_{\mathrm{ex}}\left(r \leq r_{0}\right) & =r^{2} / 1000-1+999 / 4000 \\ u_{\mathrm{ex}}\left(r \geq r_{0}\right) & =r^{2}-1\end{cases}
$$

The exact value of the flux, equal in both $\Omega_{1}$ and $\Omega_{2}$ is

$$
\nu \nabla v=2 r \mathbf{e}_{r},
$$

so that the exact value of the normal flux along the interface is $q_{\mathrm{ex}}=2 r_{0}$

We compute the approximate solution to this problem using five different meshes with increasing number of elements and the three different schemes (FEM, XFEM, XFEM+). For each mesh and each scheme we compute the relative error in terms of the flux at the interface as

$$
e=\frac{\left|q_{\mathrm{ex}}-q_{h}\right|}{\left|q_{\mathrm{ex}}\right|},
$$

where $q^{h}$ is the approximate flux, computed on the interface $\Gamma$ on the side of $\Omega_{2}$. We then plot in Figure 9 the evolution of that error with the size of the elements. This figure shows that the only method that converges with the size 


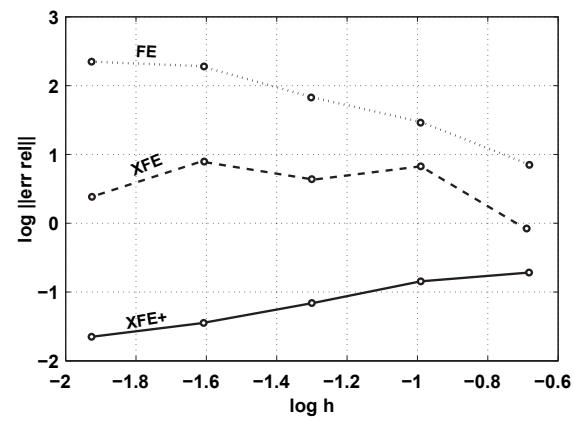

Figure 9: Convergence of the relative error of the flux (not the flux jump) with the size of the elements for the FEM (dotted line), the XFEM (dashed line) and the XFEM+ (solid line).

of the elements is the XFEM+. The convergence rate varies between about 0.5 and 1, although it is difficult to estimate the theoretical rate of convergence that the curve should follow. Interestingly, it can be noted that, in terms of the flux, the solution of the standard FEM deteriorates as the elements get smaller. Note, however, that we are measuring here only the accuracy of the flux close to the interface. Obviously the error in terms of the global energy norm gets smaller as the mesh is refined. The errors of the XFEM seem, at least for this example, to be independent of the mesh size and are maintained within the same order as $h$ gets smaller.

\section{CONCLUSIONS}

In this paper, we have presented a modification of the XFEM for bi-phasic diffusion problems, that improves the evaluation of the fluxes in the vicinity of the interface. It requires the solution of a mixed problem and enforces weakly the continuity of the fluxes over the interface. The stability of the mixed problem has been discussed based on the Chapelle-Bathe numerical test, which was passed satisfactorily. For a very limited loss of accuracy in terms of global energy norm (in the worst case found in the examples, the undervaluation is of $0.5 \%$, in the rest of cases, below the resolution of the four significant digits display), the examples presented in the paper showed that our method behaves extremely well in terms of the evaluation of the flux over the interface. The use of this improved method seems extremely appealing for the wide range of problems where the evolution of the interface is controlled by the fluxes along that interface. Note that the errors in the evaluation of the fluxes with the FEM and XFEM are particularly important when there is a large difference in properties between the two domains, so that our approach is particularly interesting in those cases (in the examples in this paper we have considered ratios of properties of 1000). 


\section{A Analytical study of stability}

The numerical demonstration of stability presented in section 6 , based on the ideas of [29] is here complemented by a theoretical result. This proposition states that for the proposed formulation, the LBB condition is proven to hold. This result requires some assumptions that are not obvious to guarantee but that, in practice, are fulfilled.

The proof is based on one idea already introduced in the numerical test. For the selected spaces, it is shown that any function $\mu$ in the Lagrange multipliers space, $\tilde{V}_{H}$ can be recovered as the jump of the normal flux of some function $v$ in $V_{X}$. Moreover, it is also shown that the norm of $v$ is bounded by above by the norm of $\mu$.

First, these properties are stated in an element-wise format.

Lemma A.1 (Element-wise property) Let $\Omega^{e}$ be one linear triangular element crossed by the interface $\Gamma$. The restriction of $\Gamma$ to $\Omega^{e}$ is denoted $\Gamma^{e}$. The nodes of $\Omega^{e}$ are denoted $P_{1}, P_{2}$ and $P_{3}$, choosing the order such that $P_{1}$ and $P_{2}$ are on the same side of the interface. As classically done in XFEM, we assume that $\exists \epsilon>0$ such that $\left|\Gamma^{e}\right|>\epsilon$. The restrictions of the functional spaces $V_{X}$ and $\tilde{V}_{H}$ to $\Omega^{e}$ and $\Gamma^{e}$ are denoted $V_{X}^{e}$ and $\tilde{V}_{H}^{e}$, with their respective $\mathcal{L}_{2}$ norms $\|v\|_{V_{X}^{e}}^{2}=\int_{\Omega^{e}} v^{2} d \Omega$ and $\|\mu\|_{\tilde{V}_{H}^{e}}^{2}=\int_{\Gamma^{e}}^{X} \mu^{2} d \Gamma$. The standard FE shape function corresponding to the node $P_{1}$ is denoted $N_{1}$, and the ridge function, defined at equation (16), is denoted $R$.

Then $\exists \alpha>0$ such that $\forall \mu \in \tilde{V}_{H}^{e}, \exists v \in \operatorname{span}\left\{N_{1}, R N_{1}\right\} \subset V_{X}^{e}$ (i.e. describing $v$ with the d.o.f. corresponding to $P_{1}$ only) verifying

$$
\begin{aligned}
& \text { 1. } \llbracket \nu \nabla v \cdot \mathbf{n} \rrbracket=\mu ; \\
& \text { 2. }\|v\|_{V_{X}^{e}} \leq \alpha\|\mu\|_{\tilde{V}_{H}^{e}} .
\end{aligned}
$$

Let us consider a function $v=u_{1} N_{1}+a_{1} N_{1} R \in \operatorname{span}\left\{N_{1}, R N_{1}\right\}$, where $u_{1}$ and $a_{1}$ are two scalar values. Let us denote by $\gamma_{1}$ and $\gamma_{2}$ the jumps of the normal fluxes corresponding to $N_{1}$ and $R N_{1}$, namely

$$
\gamma_{1}:=\llbracket \nu \nabla N_{1} \cdot \mathbf{n} \rrbracket \text { and } \gamma_{2}:=\llbracket \nu \nabla N_{1} R \cdot \mathbf{n} \rrbracket .
$$

Note that $\gamma_{1}$ is a constant function on $\Gamma^{e}$, which vanishes only if $\nabla N_{1}$ is orthogonal to $\mathbf{n}$, that is in the case of being $\Gamma^{e}$ perpendicular to the side $P_{2} P_{3}$ of the element. In this case, the node $P_{1}$ has to be replaced by $P_{2}$. Function $\gamma_{2}$ is linear (not constant) and therefore $\left\{\gamma_{1}, \gamma_{2}\right\}$ is a basis of $\tilde{V}_{H}^{e}$. Thus, varying the coefficients $u_{1}$ and $a_{1}$, the jump of $v$, may reproduce any function $\mu \in \tilde{V}_{H}^{e}$, that is

$$
\mu=u_{1} \gamma_{1}+a_{1} \gamma_{2}=\llbracket \nu \nabla v \cdot \mathbf{n} \rrbracket .
$$

Moreover, being both $v$ and $\mu$ represented by the coefficients $\left[u_{1} a_{1}\right]$ in the bases $\left\{N_{1}, N_{1} R\right\}$ and $\left\{\gamma_{1}, \gamma_{2}\right\}$ respectively, their norms are expressed as

$$
\|v\|_{V_{X}^{e}}^{2}=\left[u_{1} a_{1}\right] \mathbf{M}_{v}\left[u_{1} a_{1}\right]^{\top} \text { and }\|\mu\|_{\tilde{V}_{H}^{e}}^{2}=\left[u_{1} a_{1}\right] \mathbf{M}_{\mu}\left[u_{1} a_{1}\right]^{\top},
$$


being $\mathbf{M}_{v}$ and $\mathbf{M}_{\mu} 2 \times 2$ mass matrices with generic terms

$$
\left[\mathbf{M}_{v}\right]_{i j}=\int_{\Omega^{e}} N_{1}^{2} R^{i+j-2} d \Omega \text { and }\left[\mathbf{M}_{\mu}\right]_{i j}=\int_{\Gamma^{e}} \gamma_{i} \gamma_{j} d \Gamma \text { for } i, j=1,2
$$

The second part of the statement is readily proved by taking $\alpha$ equal to $\tilde{\alpha}_{\max }$, the maximum eigenvalue of the following generalized eigenvalue problem.

$$
\mathbf{M}_{v} \mathbf{q}=\tilde{\alpha} \mathbf{M}_{\mu} \mathbf{q}
$$

The fact that this eigenvalue is bounded from below (it cannot be indefinitely small) is guaranteed by the fact that the elements are regular enough and the minimum length of the interface inside the element is also limited.

This bounding property holds for a given value of $H$. It is necessary to check also the tendency of $\alpha$ in the limit case, that is when $H$ tends to 0 . Note that, with respect to the characteristic element size, $H, \mathbf{M}_{v}$ scales with $H^{2}$ and $\mathbf{M}_{\mu}$ scales with $H$. Therefore, $\alpha$ is expected to scale with $H$. This is the right tendency for the final result shown in Theorem A.4 because it has to be guaranteed that $k$ is larger than a given value and $k$ is taken as the inverse of $\alpha$. Thus, taking $k$ equal to the inverse of the $\alpha$ corresponding to $H_{\max }$ (the largest possible element size) we are in the safe side.

The next proposition states that the decay of a function $v$ defined in an element $\Omega^{e}$ is fast enough to control the propagation of the norm into the neighbor elements.

Lemma A.2 (Bound of the norm propagated to the neighbor elements) Let $\Omega^{e}$ and $\Omega^{e+1}$ be two contiguous elements crossed by the interface and $P_{1} P_{2}$ and $P_{3}$ the nodes of $\Omega^{e}$. Then, $\exists \beta>0$ such that, for any $v$ defined by the d.o.f. of $\Omega^{e}$,

$$
v \in \operatorname{span}\left\{N_{i}, R N_{i}\right\} \quad i=1,2,3
$$

it holds that

$$
\|v\|_{V_{X}^{e+1}} \leq \beta\|v\|_{V_{X}^{e}}
$$

Let us denote $P_{1}$ and $P_{3}$ the common nodes to $\Omega^{e}$ and $\Omega^{e+1}$, being $P_{2}$ the third node in $\Omega^{e}$, see figure 10. $P_{1}$ is selected such that it is on the same side of the interface as $P_{2}$. The third node in $\Omega^{e+1}$ is denoted as $P_{4}$ as shown in Figure 10. In order to define a mapping transforming $\Omega^{e}$ into $\Omega^{e+1}$ we introduce the following: let $O$ be the intersection point of straight lines $P_{1} P_{3}$ and $P_{2} P_{4}$, $\gamma=\frac{\left|O P_{4}\right|}{\left|O P_{2}\right|}$ and $\theta$ the angle between $O P_{1}$ and $O P_{2}$. Then the affinity, $A$, of axis $P_{1} P_{3}$ following the direction of $P_{2} P_{4}$ and ratio $-\gamma$, maps $\Omega^{e}$ into $\Omega^{e+1}$. Selecting the proper coordinate axis, the Jacobian of the affinity is

$$
J^{A}=\left(\begin{array}{cc}
-\gamma & -\gamma \cot \theta \\
0 & 1
\end{array}\right)
$$


and the absolute value of its determinant yields $\left|J^{A}\right|=\gamma$.

$$
\begin{aligned}
\|v\|_{V_{X}^{e+1}}^{2} & =\int_{\Omega^{e+1}}\left(u_{1} N_{1}+u_{3} N_{3}+a_{1} R N_{1}+a_{3} R N_{3}\right)^{2} d \Omega \\
& =\left.\int_{\Omega^{e}}\left(u_{1} N_{1}+u_{3} N_{3}+a_{1} R N_{1}+a_{3} R N_{3}\right)^{2}\right|_{\Omega^{e+1}} \circ A\left|J^{A}\right| d \Omega(46)
\end{aligned}
$$

Note that due to the similarity of the shape functions $\left.N_{i}\right|_{\Omega^{e+1}} \circ A=\left.N_{i}\right|_{\Omega^{e}}$ for $i=1,3$.

Let us denote $\tilde{\Gamma}$ as the inverse mapping of the interface $\Gamma^{e+1}$ into $\Omega^{e}$, namely $\tilde{\Gamma}=A^{-1}\left(\Gamma^{e+1}\right)$. Note that the ridge function associated with $\tilde{\Gamma}, \tilde{R}$, is such that $\tilde{R}=\left.R\right|_{\Omega^{e+1}} \circ A$. The equation (46) can be rewritten as

$$
\|v\|_{V_{X}^{e+1}}^{2}=\int_{\Omega^{e}}\left(u_{1} N_{1}+u_{3} N_{3}+a_{1} \tilde{R} N_{1}+a_{3} \tilde{R} N_{3}\right)^{2} \gamma d \Omega .
$$

Recall that

$$
\|v\|_{V_{X}^{e}}^{2}=\int_{\Omega^{e}}\left(u_{1} N_{1}+u_{2} N_{2}+u_{3} N_{3}+a_{1} R N_{1}+a_{2} R N_{2}+a_{3} R N_{3}\right)^{2} d \Omega(48)
$$

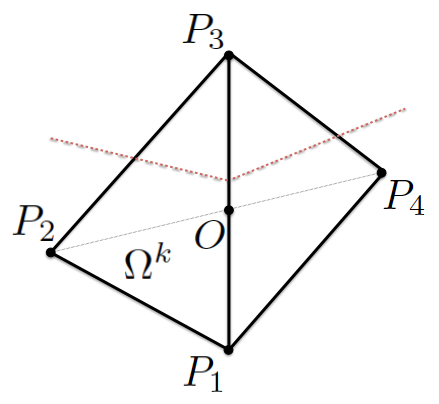

Figure 10: Illustration of two contiguous elements $\Omega^{e}$ and $\Omega^{e+1}$ for lemma A.2

The following lemma is a straightforward corollary of the two previous lemmas.

Lemma A.3 (Extension of lemma 1 to the entire space assuming the limited propagation of the Under the hypotheses of the previous lemmas, $\exists \alpha>0$ such that $\forall \mu \in \tilde{V}_{H}$, $\exists v \in V_{X}$ verifying
1. $\llbracket \nu \nabla v \cdot \mathbf{n} \rrbracket=\mu$;
2. $\|v\|_{V_{X}} \leq \alpha\|\mu\|_{\tilde{V}_{H}}$. 
The following theorem is a direct consequence of the previous lemma and guarantees the fulfillment of the LBB condition.

Theorem A.4 Under the assumptions of regularity of the mesh and of local length of the interface, $\exists k>0$ such that

$$
\inf _{\mu \in \tilde{V}_{H}} \sup _{w \in V_{H}} \frac{b(\mu, w)}{\|\mu\|_{\tilde{V}_{H}}\|w\|_{V_{X}}} \geq k
$$

For a given $\mu$, take $v$ as indicated in Lemma A.3. Then

$$
b(\mu, w)=\int_{\Gamma} \mu^{2} d \Gamma=\|\mu\|_{\tilde{V}_{H}}^{2}
$$

and

$$
\frac{b(\mu, v)}{\|\mu \mid\|_{\tilde{V}_{H}}\|v\|_{V_{X}}}=\frac{\left.\|\mu\|\right|_{\tilde{V}_{H}}}{\|v\|_{V_{X}}}
$$

Since $\|v\|_{V_{X}} \leq \alpha\|\mu\|$, taking $k=1 / \alpha$ the proposition follows.

This is equivalent to satisfy the LBB condition and guarantees the stability of the scheme.

Support from Ministerio de Educación y Ciencia, Grant DPI2011-27778C02-02 is deeply acknowledged.

\section{References}

[1] Goldstein D, Handler R, Sirovich L. Modeling a no-slip flow boundary with an external force field. J. Comp. Phys. 1993; 105(2):354-366, doi: 10.1006/jcph.1993.1081.

[2] Angot P, Bruneau CH, Fabrie P. A penalization method to take into account obstacles in incompressible viscous flows. Numerische Mathematik 1999; 81(4):497-520, doi:10.1007/s002110050401.

[3] Peskin CS. The immersed boundary method. Acta Numer. 2002; 11:479517, doi:10.1017/S0962492902000077.

[4] Vennat E, Aubry D, Degrange M. Collagen fiber network infiltration: permeability and capillary infiltration. Transport Porous Media 2010; 84(3):717-733, doi:10.1007/s11242-010-9537-4.

[5] Barrett JW, Elliott CM. Fitted and unfitted finite-element methods for elliptic equations with smooth interfaces. IMA J. Numer. Anal. 1987; 7(3):283-300, doi:10.1093/imanum/7.3.283.

[6] Mackinnon RJ, Carey GF. Treatment of material discontinuity in finite element computations. Int. J. Numer. Meth. Engrg. 1987; 24(2):393-417, doi:10.1002/nme.1620240209. 
[7] Pehlivanov AI, Lazarov RD, Carey GF, Chow SS. Superconvergence analysis of approximate boundary-flux calculations. Numerische Mathematik 1992; 63(1):483-501, doi:10.1007/BF01385871.

[8] Melenk JM, Babuška I. The partition of unity finite element method: basic theory and applications. Comp. Meth. Appl. Mech. Engrg. 1996; 139(14):289-314.

[9] Oden JT, Duarte CA, Zienkiewicz OC. A new cloud-based hp finite element method. Comp. Meth. Appl. Mech. Engrg. 1998; 153(1-2):117-126.

[10] Moës N, Dolbow J, Belytschko T. A finite element method for crack growth without remeshing. Int. J. Numer. Meth. Engrg. 1999; 46(1):131-150, doi: 10.1002/(SICI)1097-0207(19990910)46:1〈131::AID-NME726〉3.0.CO;2-J.

[11] Chessa J, Smolinski P, Belytschko T. The extended finite element method (XFEM) for solidification problems. Int. J. Numer. Meth. Engrg. 2002; 53(8):1959-1977, doi:10.1002/nme.386.

[12] Merle R, Dolbow J. Solving thermal and phase change problems with the eXtended finite element method. Comp. Mech. 2002; 28(5):339-350, doi: 10.1007/s00466-002-0298-y.

[13] Belytschko T, Parimi C, Moës N, Sukumar N, Usui S. Structured extended finite element methods of solids defined by implicit surfaces. Int. J. Numer. Meth. Engrg. 2003; 56(4):609-635, doi:10.1002/nme.686.

[14] Chessa J, Belytschko T. An extended finite element method for two-phase fluids: flow simulation and modeling. J. Appl. Mech. 2003; 70(1):10-17, doi:10.1115/1.1526599.

[15] Moës N, Cloirec M, Cartaud P, Remacle JF. A computational approach to handle complex microstructure geometries. Comp. Meth. Appl. Mech. Engrg. 2003; 192(28-30):3163-3177, doi:10.1016/S0045-7825(03)00346-3.

[16] Dolbow J, Fried E, Ji H. A numerical strategy for investigating the kinetic response of stimulus-responsive hydrogels. Comp. Meth. Appl. Mech. Engrg. 2005; 194(42-44):4447-4480, doi:10.1016/j.cma.2004.12.004.

[17] Duddu R, Bordas S, Chopp D, Moran B. A combined extended finite element and level set method for biofilm growth. Int. J. Numer. Meth. Engrg. 2008; 74(5):848-870, doi:10.1002/nme.2200.

[18] Legrain G, Moës N, Huerta A. Stability of incompressible formulations enriched with X-FEM. Comp. Meth. Appl. Mech. Engrg. 2008; 197(2124):1835-1849, doi:10.1016/j.cma.2007.08.032.

[19] Zlotnik S, Díez P. Hierarchical X-FEM for n-phase flow (n>2). Comp. Meth. Appl. Mech. Engrg. 2009; 198(30-32):2329-2338, doi:10.1016/j.cma.2009. 02.025 . 
[20] Fries TP. The intrinsic XFEM for two-fluid flows. Int. J. Numer. Meth. Fluids 2009; 60(4):437-471, doi:10.1002/fld.1901.

[21] Cottereau R, Díez P, Huerta A. Modeling, with a unified level-set representation, of the expansion of a hollow in the ground under different physical phenomena. Comp. Mech. 2010; 46(2):315-327, doi:10.1007/ s00466-009-0443-y.

[22] Knapen A, Poesen J, Govers G, Gyssels G, Nachtergaele J. Resistance of soils to concentrated flow erosion: a review. Earth-Sci. Rev. 2007; 80(12):75-109, doi:10.1016/j.earscirev.2006.08.001.

[23] Hansbo A, Hansbo P. An unfitted finite element method, based on Nitsche's method, for elliptic interface problems. Comp. Meth. Appl. Mech. Engrg. 2002; 191(47-48):5537-5552, doi:10.1016/S0045-7825(02)00524-8.

[24] Harari I, Dolbow J. Analysis of an efficient finite element method for embedded interface problems. Comp. Mech. 2010; 46:205-211, doi:10.1007/ s00466-009-0457-5.

[25] Zunino P, Cattaneo L, Colciago CM. An unfitted interface penalty method for the numerical approximation of contrast problems. Appl. Numer. Math. 2011; doi:10.1016/j.apnum.2011.06.005.

[26] Ji H, Dolbow JE. On strategies for enforcing interfacial constraints and evaluating jump conditions with the extended finite element method. Int. J. Numer. Meth. Engrg. 2004; 61(14):2508-2535, doi:10.1002/nme.1167.

[27] Moës N, Béchet E, Tourbier M. Imposing Dirichlet boundary conditions in the extended finite element method. Int. J. Numer. Meth. Engrg. 2006; 67(12):1641-1669, doi:10.1002/nme.1675.

[28] Béchet E, Moës N, Wohlmuth B. A stable Lagrange multiplier space for stiff interface conditions within the extended finite element method. Int. J. Numer. Meth. Engrg. 2009; 78(8):931-954, doi:10.1002/nme.2515.

[29] Chapelle D, Bathe KJ. The inf-sup test. Comp. \& Struct. 1993; 47(45):537-545.

[30] Bathe KJ. The inf-sup condition and its evaluation for mixed finite element methods. Comp. \& Struct. 2001; 79(2):243-252.

[31] Kim TY, Dolbow J, Laursen T. A mortared finite element method for frictional contact on arbitrary interfaces. Comp. Mech. 2007; 39(3):223235, doi:10.1007/s00466-005-0019-4.

[32] Crank J. Free and moving boundary problems. Oxford University Press, 1984. 
[33] Unverdi SO, Tryggvason G. A front-tracking method for viscous, incompressible, multi-fluid flows. J. Comp. Phys. 1992; 100(1):25-37, doi: 10.1016/0021-9991(92)90307-K.

[34] Gorczyk W, Gerya TV, Connolly JAD, Yuen DA, Rudolph M. Large-scale rigid-body rotation in the mantle wedge and its implications for seismic tomography. Geochem. Geophys. Geosyst. 2006; 7(5). Doi:10.1029/2005GC001075.

[35] Jan YJ. A cell-by-cell thermally driven mushy cell tracking algorithm for phase-change problems. Comp. Mech. 2007; 40(2):201-216, doi:10.1007/ s00466-006-0098-x.

[36] Sethian JA. Level Set Methods and Fast Marching Methods: Evolving Interfaces in Computational Geometry, Fluid Mechanics, Computer Vision, and Materials Science. Cambridge University Press: Cambridge, U.K., 1999.

[37] Osher S, Fedkiw R. Level set methods: an overview and some recent results. J. Comp. Phys. 2001; 169(2):463-502, doi:10.1006/jcph.2000.6636.

[38] Osher S, Sethian JA. Fronts propagating with curvature-dependent speed: Algorithms based on Hamilton-Jacobi formulations. J. Comp. Phys. 1988; 79(1):12-49, doi:10.1016/0021-9991(88)90002-2.

[39] Sussman M, Smereka P, Osher S. A level set approach for computing solutions to incompressible two-phase flow. J. Comp. Phys. 1994; 114(1):146159, doi:10.1006/jcph.1994.1155.

[40] Mulder W, Osher S, Sethian JA. Computing interface motion in compressible gas dynamics. J. Comp. Phys. 1992; 100(2):209-228, doi:10.1016/ 0021-9991(92)90229-R.

[41] Karlsen KH, Lie KA, Risebro NH. A fast marching method for reservoir simulation. Comp. Geosci. 2000; 4(2):185-206, doi:10.1023/A: 1011564017218.

[42] Nielsen LK, Li H, Tai XC, Aanonsen SI, Espedal M. Reservoir description using a binary level set model. Comp. Visu. Sci. 2008; doi:10.1007/ s00791-008-0121-1.

[43] Zlotnik S, Díez P, Fernández M, Vergés J. Numerical modelling of tectonic plates subduction using X-FEM. Comp. Meth. Appl. Mech. Engrg. 2007; 196(41-44):4283-4293, doi:10.1016/j.cma.2007.04.006.

[44] Zlotnik S, Fernández M, Díez P, Vergés J. Modelling gravitational instabilities: slab break-off and Rayleigh-Taylor diapirism. Pure Appl. Geophys. 2008; 165:1-20, doi:10.1007/s00024-004-0386-9.

[45] Sethian JA, Popovici AM. 3-D traveltime computation using the fast marching method. Geophys. 1999; 64(2):516-523, doi:10.1190/1.1444558. 
[46] Ito K, Kunisch K, Li Z. Level-set function approach to an inverse interface problem. Inverse Prob. 2001; 17(5):1225-1242, doi:10.1088/0266-5611/17/ $5 / 301$.

[47] Burger M. A level set method for inverse problems. Inverse Prob. 2001; 17(5):1327-1355, doi:10.1088/0266-5611/17/5/307.

[48] Burger M, Osher SJ. A survey of level set methods for inverse problems and optimal design. Europ. J. Appl. Math. 2005; 16(2):263-301, doi:10.1017/ S0956792505006182.

[49] Cordero F, Díez P. XFEM+: una modificación de XFEM para mejorar la precisión de los flujos locales en problemas de difusión con conductividades muy distintas. Revista Internacional Métodos Numéricos para Cálculo y Diseño en Ingeniería 2010; 26(2):121-133.

[50] Cottereau R, Díez P. Numerical modeling of erosion using an improvement of the extended finite element method. Europ. J. Environmental Civil Engrg. 2011; 15(8):1187-1206, doi:10.3166/EJECE.15.1187-1206.

[51] Legrain G, Allais R, Cartraud P. On the use of the extended finite element method with quadtree/octree meshes. Int. J. Numer. Meth. Engrg.. 2011; 86:717-743.

[52] Soghrati S, Aragón AM, Duarte CA, Geubelle PH. An interface-enriched generalized FEM for problems with discontinuous gradient fields. Int. J. Numer. Meth. Engrg. 2012; 89(8):991-1008.

[53] Brezzi F. On the existence, uniqueness and approximation of saddlepoint problems arising from lagrangian multipliers. Revue Française d'Automatique, Informatique et Recherche Opérationnelle 1974; 8(2):129151. 